\title{
Lymphocyte dynamics: caution in interpreting BAL numbers
}

\author{
Reinhard Pabst, Thomas Tschernig
}

\begin{abstract}
In various allergic and inflammatory lung diseases the number and subset composition of lymphocytes in the bronchoalveolar lavage (BAL) fluid are taken as indicators of the state of the disease. The number of lymphocytes in the BAL fluid depends on three main parameters: (1) entry into the bronchoalveolar space from the different compartments of the lung, (2) persistence in the bronchoalveolar space which is modified by the rate of local proliferation and apoptosis, and (3) exit into the draining bronchial lymph node via the lymphatic system. In healthy individuals lymphocytes in the BAL fluid seem to be a stable pool: each day there is hardly any entry, local cell division or cell death and few lymphocytes emigrate from this compartment. In contrast, during inflammatory, toxic and allergic reactions all parameters can increase rapidly with more lymphocytes entering, proliferating and/ or undergoing apoptosis locally. Very little is known about factors such as cytokines and chemokines which may regulate these
\end{abstract}

Centre of Anatomy 4120 ,

Hannover,

D-30623 Hannover,

Germany

R Pabst

Correspondence to: Professor Dr R Pabst. parameters. When interpreting data on lymphocyte numbers in patients, lymphocyte dynamics in the bronchoalveolar space have to be considered, and in the future it may be possible to manipulate these lymphocyte fluxes for therapeutic purposes.

(Thorax 1997;52:1078-1080)

Keywords: bronchoalveolar lavage, lymphocytes, origin, proliferation, apoptosis, lymphocyte dynamics.

Bronchoalveolar lavage (BAL) has become a routine clinical procedure applied with increasing frequency. Due to a minimum of side effects it can even be applied in volunteers, in patients with allergies such as asthma, and during intensive care. Recommendations for a standardised procedure have been published. ${ }^{1}$ Lymphocytes constitute about $10 \%$ of all cells in the BAL fluid of healthy adults. In sarcoidosis and diffuse panbronchiolitis, after allergen provocation in asthma, or in interstitial lung diseases and idiopathic eosinophilic pneumonia lymphocytes are increased or $\mathrm{T}$ cell subsets are found in a composition different from that in control subjects. ${ }^{2-4}$

One basic prerequisite for a meaningful interpretation of data on lymphocytes in BAL fluid is that not only the percentage of lymphocytes or their subsets but also the total number per volume are documented in all studies. In most situations only a single lavage can be performed in an individual patient. Thus, the data are like a photographic snapshot - only indicative of pathophysiological events if it portrays a stable condition rather than one stage of a disease process. As lymphocytes continuously patrol through nearly all organs of the body, leaving the blood to traverse the organ parenchyma and returning to the blood via the lymphatic system, the number of lymphocytes in the lung also depends on the rate of entry of lymphocytes into the bronchoalveolar space, the rate of exit, and the fate of the lymphocytes within the bronchoalveolar space (fig 1).

Scientific basis

LYMPHOCYTE MIGRATION INTO THE LUNG Lymphocytes are found in different compartments of the lung: ${ }^{5}$ the intravascular
Figure 1 Schematic drawing of the entry and exit of lymphocytes in the BAL fluid. The kinetics of lymphocytes in the BAL fluid depend on factors regulating the entry from different lung compartments, the local proliferation and apoptosis, and the extent of exit from the bronchoalveolar space. 
"marginal pool", the interstitial pool, within the bronchial epithelium and the bronchial lamina propria. There is no hard evidence in experimental animals or in humans that lymphocytes migrate from all these compartments into the bronchoalveolar space. Less than $10 \%$ of the lymphocytes in the BAL fluid are B cells, and among the T cells the CD $4+$ cells outnumber the CD $8+$ cells. There are many more so-called "memory" (>85\%) than "naive" T lymphocytes in the BAL fluid, which is different from the composition of lymphocytes in other lung compartments. ${ }^{5}$ Either this subset preferentially migrates into the bronchoalveolar space or "naive" lymphocytes change their phenotype on arrival.

\section{LYMPHOCYTE PROLIFERATION}

Proliferation of lymphocytes can be measured by the uptake of DNA precursors - for example, ${ }^{3} \mathrm{H}$-thymidine $\left({ }^{3} \mathrm{H}-\mathrm{TdR}\right)$, bromodeoxy uridine (BrdU), or markers for lymphocytes in the cell cycle such as Ki67. In patients with HIV infection, lymphocytes obtained by BAL incorporated more ${ }^{3} \mathrm{H}-\mathrm{TdR}$ than those from healthy volunteers and proliferation was confined to the CD8 + population. ${ }^{6}$ Chilosi et al studied the expression of $\mathrm{Ki} 67$ on $\mathrm{T}$ lymphocytes recovered from BAL fluid and found no Ki67-labelled T lymphocytes in specimens from healthy volunteers but in patients with active sarcoidosis approximately $6 \%$ of all $\mathrm{T}$ cells were labelled.

\section{APOPTOSIS}

Programmed cell death, also called apoptosis, can be identified by the expression of CD95 (Fas). In a recent study Agostini et al ${ }^{8}$ reported that Fas antigen was weakly expressed in BAL fluid samples from control subjects but was upregulated in BAL fluid from patients with sarcoidosis. Studies on the termination of pulmonary immune reactions - for example, using sheep red blood cells as an antigen in mice revealed the role of apoptosis in the elimination of lung lymphocytes. ${ }^{9}$ In a recent study the marker bcl-2, which inhibits apoptosis resulting in prolonged cell survival, was found to occur more frequently in lymphocytic interstitial pneumonia. ${ }^{10}$ For the lymphocytes of healthy individuals neither local proliferation nor apoptosis seem to play a role in the bronchoalveolar space. In lung diseases, however, these two parameters indicate an increased lymphocyte turnover.

REMOVAL FROM THE BRONCHOALVEOLAR SPACE One long standing hypothesis was that lymphocytes from the bronchoalveolar space are transported by the mucociliary escalator into the trachea, then coughed up, swallowed, and destroyed. In healthy young pigs labelled lymphocytes obtained from the peripheral blood were instilled into the bronchoalveolar space. After a few hours they were seen in the draining bronchial lymph node. Lymphocyte subsets differed in their speed and frequency of mi- gration to the draining lymph node. ${ }^{11}$ From an immunological point of view it is relevant that lymphocytes from the bronchoalveolar space return to the lymphoid system where individual clones can expand and the lymphocytes can migrate to other organs. No regulatory factors are known so far which influence the migration of lymphocytes from the BAL fluid to the lymph nodes but adhesion molecules might play a role. The adhesion molecules which are known as regulatory molecules in lymphocyte migration via high endothelial venules in lymph nodes - for example, selectins, integrins and molecules of the super Ig family - are obviously not important in the adhesion of lymphocytes to pulmonary endothelium.

Very little is known of the origin of lymphocytes in the BAL fluid and the route from the blood to the bronchoalveolar space (fig 1). Thoracic duct lymphocytes were labelled in vitro with FITC in rats and injected intravenously. A labelling index of $>1 \%$ was found in all lymphoid organs studied and remained in the blood for several days. However, in the bronchoalveolar space no FITC + lymphocytes were found. ${ }^{5}$ When pigs were immunised orally with a lung pathogenic bacterium they were protected in a subsequent challenge and the numbers of CD $4+$ and CD8 + lymphocytes increased in parallel in the bronchoalveolar space. The bronchoalveolar space is therefore included in the integrated mucosal system.

In allergic reactions provoked by local antigens instillation induced an increased number of lymphocytes in the bronchoalveolar space. As recently summarised, ${ }^{4}$ different gases (such as $\mathrm{SO}_{2}, \mathrm{NO}_{2}$, and ozone) as well as lipopolysaccharide instillation $^{12}$ in the airways of healthy volunteers all resulted in increased numbers of lymphocytes in the BAL fluid. The rapidity of the effect (within a few hours) makes it likely that these increases are due to enhanced entry into the bronchoalveolar space because enhanced local proliferation would hardly be so quick, and exit is also very low under normal circumstances. It is not known whether the toxic gases themselves attract the lymphocytes from the different compartments. Local effects of interleukins and chemokines during immune reactions could also be candidates for opening the door for lymphocytes to enter the bronchoalveolar space. ${ }^{4}$

\section{Therapeutic potential and conclusions}

It should always be kept in mind that increased numbers of lymphocytes in BAL fluid could be caused by increased influx, increased local proliferation, reduced apoptosis or decreased efflux, or even a combination of all of these parameters. Minor differences in the influx of lymphocytes could cause major increases in lymphocyte numbers over time resulting in an accumulation in the bronchoalveolar space. When more data are available on the role of adhesion molecules, cytokines, and chemokines as factors influencing lymphocytes in their speed of entry, persistence in the bronchoalveolar space, and rate of exit from the bronchoalveolar space, new therapeutic 
strategies can be planned. Many more kinetic data are needed before the lymphocyte fluxes will be understood well enough to form a hypothesis on the pathophysiology of many lung diseases.

1 Klech H, Pohl W, eds. Technical recommendations and guidelines for bronchoalveolar lavage (BAL). Eur Respir $\mathcal{F}$ 1989;2:561-85.

2 Semenzato G. Immunology of interstitial lung diseases: cellular events taking place in the lung of sarcoidosis, hypersensitivity pneumonitis and HIV infection. Eur Respir f 1991;4:94-102.

3 Corrigan CJ, Kay AB. The lymphocyte in asthma. In: Busse WW, Holgate SF, eds. Asthma and rhinitis. Boston: Blackwell Science, 1995: 450-64.

4 Pabst R, Tschernig T. What is known about the life span and fate of lymphocytes in the bronchoalveolar space? In: Husband AJ, ed. Mucosal solutions - advances in mucosal immunology. Sydney University, 1997 (in press)

5 Pabst R, Tschernig T. Lymphocytes in the lung: an often neglected cell. Numbers, characterization and compartmentalization. Anat Embryol 1995;192:293-9.
6 Spain BA, Soliman DM, Sidner RA, Twigg HL. Enhanced proliferation and IL-2 secretion by lung lymphocytes from HIV-infected subjects. Am F Physiol 1995;269:L498-506.

7 Chilosi M, Menestrina F, Capelli P, Montagna L, Lestan $\mathrm{M}$, Pizzolo G, et al. Immunohistochemical analysis of sarcoid granulomas. Evaluation of Ki67 + and interleukin$1+$ cells. Am F Pathol 1988;131:191-8.

8 Agostini C, Zambello R, Sancetta R, Cerutti A, Milani A, Tassinari C, et al. Expression of tumor necrosis factorreceptor superfamily members by lung $\mathrm{T}$ lymphocytes in interstitial lung disease. Am f Respir Crit Care Med 1996; 153:1359-67.

9 Buechner-Maxwell VA, Milik AM, Sonstein J, Seitzman GD, Beals TF, Curtis JL. Apoptosis contributes to lung lymphocyte elimination in the murine response to intratracheal sheep erythrocytes. Am $\mathcal{F}$ Respir Crit Care Med 1995;151:A234.

10 Kaan PM, Hegele RG, Hayashi S, Hogg JC. Expression of bcl-2 and Epstein-Barr virus LMP-1 in lymphocytic interstitial pneumonia. Thorax 1997;52:12-6.

11 Pabst R, Binns RM. Lymphocytes migrate from the bronchoalveolar space to regional bronchial lymph nodes. $A m \mathcal{f}$ alveolar space to regional bronchial ly
Respir Crit Care Med 1995;151:495-9.

12 Sandström T, Bjermer L, Rylander R. Lipopolysaccharide (LPS) inhalation in healthy subjects increases neutrophils, (LPS) inhalation in healthy subjects increases neutrophils, age fluid. Eur Respir f 1992;5:992-6. 